\title{
Incompletude dos dados do programa Hiperdia em Unidades Básicas de Saúde em
}

\author{
Marabá, Pará \\ Incomplete data from the Hiperdia program in Primary Healthcare Centre in Marabá, Pará \\ Falta de datos del programa Hiperdia en Unidades Básicas de Salud en Marabá, Pará
}

Recebido: 08/07/2021 | Revisado: 17/07/2021 | Aceito: 21/07/2021 | Publicado: 28/07/2021

\author{
Matheus Sodré de Araújo \\ ORCID: https://orcid.org/0000-0003-1947-361X \\ Universidade do Estado do Pará, Brasil \\ E-mail: matheus.araujo@aluno.uepa.br \\ Nathália Lima Costa \\ ORCID: https://orcid.org/0000-0002-1763-4785 \\ Universidade do Estado do Pará, Brasil \\ E-mail: nathaliaalc884@gmail.com \\ Kellice Feitosa de Araújo \\ ORCID: https://orcid.org/0000-0003-2214-0824 \\ Universidade do Estado do Pará, Brasil \\ E-mail:kellice.araujo@aluno.uepa.br \\ Ana Karoline Brito de Oliveira \\ ORCID: https://orcid.org/0000-0002-3610-7454 \\ Universidade do Estado do Pará, Brasil \\ E-mail: ana.koliveira@aluno.uepa.br \\ Katiane da Costa Cunha \\ ORCID: https://orcid.org/0000-0001-5361-5090 \\ Universidade do Estado do Pará, Brasil \\ E-mail: katiane.cunha@uepa.br
}

\begin{abstract}
Resumo
O programa Hiperdia é fundamental para que se ocorra o rastreamento, prevenção e tratamento da Hipertensão Arterial e da Diabetes Mellitus no Brasil. Para isso, é utilizada uma ficha de cadastramento com dados do paciente assistido pelo programa que são enviados ao Ministério da Saúde. Contudo, percebe-se que algumas fichas não contêm dados preenchidos em sua totalidade dentro das Unidades Básicas de Saúde (UBS). Por isso, o objetivo deste estudo é analisar a incompletude das fichas do Hiperdia em UBs na cidade de Marabá-PA. Trata-se de um estudo observacional transversal, realizado em três UBSs. Foram analisadas 305 fichas cadastrais verificando seu índice de incompletude com o auxílio de um questionário online para coleta de dados. Em seguida, o programa EpiInfo foi utilizado para medir o grau de confiança e a tabulação dos dados foi feita no programa Microsoft Excel. A incompletude das categorias que compõem a ficha de cadastro recebeu critérios de classificação que variaram de "excelente" até "muito ruim", sendo consideradas excelentes as categorias que possuíam menor índice de incompletude. Verificou-se que grande parte das categorias foram classificadas com "ruim" ou "muito ruim". Tal resultado demonstra que o emprego do programa Hiperdia apresenta fragilidades na cidade de Marabá. Como consequência, a população da cidade que depende do programa acaba não tendo um acesso ideal à saúde, visto que o repasse incompleto dos seus dados ao Ministério da Saúde dificulta a criação de estratégias de saúde e diminui a oferta de medicamentos para os pacientes do município.

Palavras-chave: Sistemas de informação em saúde; Hipertensão; Diabetes Mellitus; Sistema Único de Saúde; Notificação de doenças.
\end{abstract}

\begin{abstract}
The Hiperdia program is fundamental for the screening, prevention, treatment of Arterial Hypertension and Diabetes Mellitus in Brazil. For that, a registration form with data from the patient assisted by the program is used, which will be sent to the Ministry of Health. However, it is clear that some forms do not contain data filled in their entirety within the Primary Healthcare Centre (PHC). Therefore, the objective of this study is to analyze the incompleteness of the Hiperdia files in PHCs in the city of Marabá-PA. This is a cross-sectional observational study, carried out in three PHCs in Marabá in 2020. 305 registration forms were analyzed, checking their incompleteness index with the help of an online questionnaire for data collection. Then, the EpiInfo program was used to measure the degree of confidence and the data were tabulated using the Microsoft Excel program. The incompleteness of the categories that make up the registration form received classification criteria that ranged from "excellent" to "very poor", being considered excellent those categories that had the lowest incompleteness index. It was found that most of the categories were classified as "poor" or "very poor". This result shows that the use of the Hiperdia program presents weaknesses in the city of Marabá. As a result of, the population of the city that depends on the program ends up not having ideal access to health, since the
\end{abstract}


incomplete transfer of their data to the Ministry of Health makes it difficult to create health strategies and reduces the supply of drugs to patients in the municipality.

Keywords: Health information systems; Hypertension; Diabetes Mellitus; Unified Health System; Disease notification.

\section{Resumen}

El programa Hiperdia es fundamental para el seguimiento, la prevención y el tratamiento de la hipertensión arterial y la diabetes mellitus en Brasil. Para ello, se utiliza un formulario de registro con datos del paciente atendido por el programa, los cuales son enviados al Ministerio de Salud. Sin embargo, es claro que algunos formularios no contienen datos completamente cumplimentados dentro de las Unidades Básicas de Salud (UBS). Por tanto, el objetivo de este estudio es analizar la incompletitud de los registros de Hiperdia en las UB de la ciudad de Marabá-PA. Se trata de un estudio observacional transversal realizado en tres SBU. Se analizaron 305 formularios de registro, verificando su índice de incompletitud con la ayuda de un cuestionario en línea para la recolección de datos. Luego, se utilizó el programa EpiInfo para medir el grado de confianza y los datos se tabularon en Microsoft Excel. La incompletitud de las categorías que componen el formulario de registro recibió criterios de clasificación que iban de "excelente" a "muy mala", considerándose excelentes las categorías que tenían la menor tasa de incompletitud. Se encontró que la mayoría de las categorías se clasificaron como "malas" o "muy malas". Este resultado demuestra que el uso del programa Hiperdia tiene debilidades en la ciudad de Marabá. Como resultado, la población de la ciudad que depende del programa termina sin tener un acceso ideal a la salud, ya que la transferencia incompleta de sus datos al Ministerio de Salud dificulta la creación de estrategias de salud y reduce la oferta de medicamentos para pacientes del municipio.

Palabras clave: Sistemas de información en salud; Hipertensión; Diabetes mellitus; Sistema único de Salud; Notificación de enfermedades.

\section{Introdução}

O Hiperdia é um programa instituído pelo Ministério da Saúde, em 2001, no contexto do Plano de Reorganização da Atenção à Hipertensão Arterial e Diabetes Mellitus (Ministério da Saúde, 2017). O programa visa oferecer assistência, prevenção e promoção de saúde a partir de planos educacionais e terapêuticos ( Feitosa \& Pimentel, 2016; Sousa \& Costa, 2020). A importância do programa na saúde pública brasileira deve-se ao fato da hipertensão arterial e da diabetes atingem órgãos alvos, como coração e rins (Silva, Silva, Santos \& Soares, 2020). Nesse contexto, estas doenças crônicas não transmissíveis estão diretamente associadas a diagnósticos como doenças cardiovasculares, doença renal crônica e acidente vascular cerebral (Prates, Souza, Prates, Moura, \& Carmo, 2020).

Para organizar o programa, o Ministério da Saúde criou o Sistema de Cadastramento e Acompanhamento de Hipertensos e Diabéticos (SIS-Hiperdia). Nele é possível fazer o cadastro e o acompanhamento das pessoas portadoras de doenças crônicas, sendo seus dados enviados para o Departamento de Informática do SUS (DATASUS), com o auxílio das Secretarias de Saúde estaduais e municipais. Para que o SIS-Hiperdia alcance seu objetivo, uma ficha de cadastro no programa é utilizada buscandose obter dados clínicos do paciente, fatores de risco, doenças, complicações e o tratamento utilizado por este (Ministério da Saúde, 2008) - informações que contribuem para que os gestores e que os profissionais de saúde consigam monitorar e enfrentar as doenças crônicas em questão (Soeiro et al., 2019; Sousa et al., 2019)

No entanto, percebe-se que os dados do SIS-Hiperdia não são preenchidos na ficha de cadastro em sua totalidade. Informações obrigatórias do paciente, como dados clínicos e documentos deixam de ser preenchidos e não são registrados pelo Ministério da Saúde. Como consequência, a integração de dados não ocorre dentro do Sistema Único de Saúde (SUS), o que torna mais difícil o diagnóstico da população que convive com doenças crônicas e a possibilidade de traçar estratégias de tratamento e de sensibilização (Zillmer, Schwartz, Muniz, \& de Lima, 2010). Nesse contexto, é válido salientar que o funcionamento do SUS também encontra como dificuldades as especificidades territoriais. Na região Amazônica, os estados possuem vastos territórios e alguns municípios são acessados somente por rios. Logo, o funcionamento das políticas públicas nesses locais demanda do entendimento de como essas regiões se organizam e do vivenciamento da singularidade de seus habitantes (Dolzane \& Schweickardt, 2020).

Localizado na região sudeste do estado do Pará, o município de Marabá possui 283.542 habitantes (Instituto Brasileiro de Geografia e Estatística, 2020) e possui uma área territorial de 15.128,058 km² (Instituto Brasileiro de Geografia e Estatística, 
2019). Na cidade, todas as Unidades Básicas de Saúde (UBS) realizam o cadastramento no Hiperdia, estimando-se que grande parte dos 20 mil dos cadastrados sejam pessoas idosas (Henriques, 2020). Por isso, diante da importância dos dados do Hiperdia, esse trabalho tem como objetivo analisar a qualidade com esses dados são processados, buscando verificar a completude do preenchimento das fichas de cadastro do Hiperdia dentro de UBSs.

\section{Metodologia}

Trata-se de um estudo quantitativo com delineamento observacional transversal(Lopes \& Harrington, 2015; Novaes, Duarte, Riveiro \& Santos, 2019). Neste estudo foi feita análise descritiva através da coleta de dados primários das fichas de cadastro do programa Hiperdia presente nos prontuários de pacientes das Unidades Básicas de Saúde (UBSs) Laranjeiras, Pedro Cavalcante e Hiroshi Matsuda, no município de Marabá no estado do Pará, entre os dias 02 de outubro e 17 de dezembro de 2020. O estudo obedeceu aos preceitos éticos da Resolução 466/12, tendo sido aprovado pelo Comitê de Ética em Pesquisa da Universidade do Estado do Pará, sob o parecer de n 3.891.065/2020, além de seguir os princípios do Código de Nuremberg e da Declaração de Helsinque.

As variáveis observadas foram divididas em 6 partes de acordo com a organização da ficha de cadastro do hipertenso e/ou diabético no programa Hiperdia: 1) Identificação do usuário: nome, data de nascimento, sexo, nome da mãe, nome do pai, raça/cor, escolaridade, nacionalidade, unidade federativa (UF) do município de nascimento, nome do município de nascimento, situação familiar/conjugal e número do cartão SUS; 2) Documentos gerais: Título de eleitor, Carteira de Trabalho e Previdência Social (CTPS), Cadastro de Pessoas Físicas (CPF) e Programa de Integração Social (PIS)/ Programa de Formação do Patrimônio do Servidor Público (PASEP); 3) Documentos obrigatórios: Identidade e certidão; 4) Endereço: tipo de logradouro, nome do logradouro, número, complemento, bairro, Código de Endereçamento Postal (CEP), Discagem Direta a Distância (DDD) e telefone; 5) Dados clínicos do paciente: pressão arterial sistólica, pressão arterial diastólica, cintura, peso, altura, glicemia capilar, fatores de risco e doenças concomitantes e presença de complicações; 6) Tratamento: Se medicamento ou não medicamentoso, uso de insulina e uso de outros medicamentos.

O instrumento utilizado para coleta de dados foi um questionário online, que continha duas possíveis respostas - "sim" ou "não" - para cada variável analisada, sendo organizado conforme a ficha de cadastramento do Hiperdia. Os espaços das fichas preenchidos de forma incompreensível ou em branco foram respondidos como não preenchidos.

$\mathrm{O}$ índice de incompletude foi calculado por meio da proporção do preenchimento nulo das variáveis e classificada nos seguintes graus de avaliação: excelente (até $5 \%$ ), bom ( $5 \%$ a $10 \%)$, regular (10\% a $20 \%)$, ruim $(20 \%$ a $50 \%$ ) e muito ruim $(50 \%$ a 100\%) (Melo \& Valongueiro, 2015).

A amostra foi definida por meio da análise da segunda via da ficha de cadastramento dos usuários do programa Hiperdia. Foram analisadas $305(32,76 \%)$ fichas cadastrais, superando o valor ideal de $272(29,21 \%)$ fichas com 95\% de confiança, segundo o programa Epiinfo ${ }^{\mathrm{TM}}$ 7.2.4.0, em um total de 931 fichas pertencentes as unidades de saúde estudadas. Para a análise dos dados, a tabulação foi feita utilizando o programa Microsoft Excel.

\section{Resultados}

A amostra totalizou 305 fichas de cadastro e de acordo com os dados analisados tem-se que a classificação geral do índice de incompletude é "ruim" (41,47\%), com a categoria "documentos gerais" tendo o pior índice $(81,86 \%)$ de preenchimento. Ademais, o item com pior índice de preenchimento da ficha de cadastro do hiperdia foi a "série" da categoria "CTPS" (97,05\%). A Tabela 1 o índice de incompletude e a classificação geral e das categorias alocadas dentro da ficha de cadastro. 
Tabela 1. Classificação por índice de incompletude das categorias da ficha de Cadastro ( $\mathrm{n}=305)$.

\begin{tabular}{lcc}
\hline Categorias da ficha de cadastro & Índice de incompletude (\%) & Classificação \\
\hline Todas as categorias & 41,47 & RUIM \\
& & \\
\hline Identificação do usuário & 10,36 & REGULAR \\
Documentos gerais & 81,86 & MUITO RUIM \\
Documentos obrigatórios & 58,26 & MUITO RUIM \\
Endereço & 39,71 & RUIM \\
Dados clínicos do paciente & 16,67 & REGULAR \\
Fatores de risco e doenças concomitantes & 27,12 & RUIM \\
Presença de complicações & 40,87 & RUIM \\
Tratamento & 56,94 & MUITO RUIM \\
\hline
\end{tabular}

Fonte: Autores.

De modo geral, de acordo com a Tabela 1, dentro das categorias das fichas de cadastro, "documentos gerais", “documentos obrigatórios" e "tratamento" tiveram os piores índices de preenchimento. Além disso, o melhor índice de preenchimento foi o de "identificação de usuários" (10,36\%), seguido dos “dados clínicos do paciente” (16,67\%), sendo que nenhuma das categorias apresentaram bom ou excelente preenchimento.

A Tabela 2 apresenta a classificação individual de cada componente das categorias da ficha de cadastro do Hiperdia.

Tabela 2. Índice de incompletude por itens das categorias da ficha de cadastro do Hiperdia. (n=305).

\begin{tabular}{lcc}
\hline Ficha de cadastro em sua totalidade & Incompletude (\%) & Classificação \\
\hline Identificação do usuário & 0,66 & EXCELENTE \\
Nome & 1,31 & EXCELENTE \\
Data de nascimento & 1,64 & EXCELENTE \\
Sexo & 4,59 & EXCELENTE \\
Nome da mãe & 17,38 & RUIM \\
Nome do pai & 6,89 & BOM \\
Raça/cor & 6,89 & BOM \\
Escolaridade & 3,28 & EXCELENTE \\
Nacionalidade & 11,15 & REGULAR \\
Estado de nascimento & 10,82 & REGULAR \\
Município de nascimento & 12,79 & REGULAR \\
Situação familiar conjugal & 46,89 & RUIM \\
Número do Cartão SUS & & \\
Documentos gerais & & \\
Título & 72,79 & MUITO RUIM \\
Número & 73,11 & MUITO RUIM \\
Zona & 73,11 & MUITO RUIM \\
Série &
\end{tabular}




\section{CTPS}

Número

Série

Estado de registro

Data da emissão

Número do CPF

Número PIS/PASEP

Documentos obrigatórios

$R G$

Número

Órgão emissor

Estado de registro

Data de emissão

\section{Certidão}

Tipo

Nome do cartório de registro

Livro

Folha

Termo

Emissão

Endereço

Tipo de logradouro

Nome do logradouro

Número

Complemento

Bairro

CEP

DDD

TELEFONE

Dados clínicos do paciente

Pressão sistólica

Pressão diastólica

Cintura

Peso

Altura

Glicemia capilar

Paciente em jejum/pós prandial cardiovasculares

Diabetes Mellitus 1
3,28

0,66

1,31

4,59

96,39

97,05

96,72

96,72

30,82

100

10,49

10,16

7,54

91,15

91,48

91,48

91,15

91,8

94,1

19,34

1,31

38,03

89,84

2,62

59,02

66,56

40,98

0,98

1,64

51,48

56,07

25,9

RUIM

MUITO RUIM

MUITO RUIM

MUITO RUIM

MUITO RUIM

RUIM

MUITO RUIM

EXCELENTE

REGULAR

REGULAR

BOM

MUITO RUIM

MUITO RUIM

MUITO RUIM

MUITO RUIM

MUITO RUIM

MUITO RUIM

REGULAR

EXCELENTE

RUIM

MUITO RUIM

EXCELENTE

MUITO RUIM

MUITO RUIM

RUIM

EXCELENTE

EXCELENTE

EXCELENTE

EXCELENTE

EXCELENTE

MUITO RUIM

MUITO RUIM 
Diabetes Mellitus 2

Tabagismo

Sedentarismo

Sobrepeso/Obesidade

Hipertensão arterial

Presença de complicações

Infarto agudo do miocárdio

Outras coronopatias

Acidente vascular cerebral

Pé diabético

Amputações por diabetes

Doença renal

\section{Tratamento}

Não medicamentoso/medicamentoso

Insulina

Outros
31,15

36,07

22,3

26,56

11,15

41,31

42,3

39,67

40,98

40,98

40

51,8

93,77

25,25
RUIM

RUIM

RUIM

RUIM

REGULAR

RUIM

RUIM

RUIM

RUIM

RUIM

RUIM

MUITO RUIM

MUITO RUIM

RUIM

Fonte: Autores.

Em relação aos índices de incompletude descritos na Tabela 2, todos os itens de "documentos gerais" apresentaram apenas classificação muito ruim, exceto "número de cpf” que obteve ruim. Em "documentos obrigatórios", prevaleceu a classificação "muito ruim" em todos os itens da certidão de nascimento, enquanto o "número de rg" teve classificação “excelente". Já em "tratamento" apenas o item "outros” foi classificado como "ruim", tendo os demais a classificação "muito ruim". Vale ressaltar também que em "identificação do usuário" apenas os itens "número do cartão SUS" e "nome do pai" tiveram classificação ruim, tendo os demais variado entre regular, bom e excelente, enquanto a categoria "dados clínicos do paciente" teve todos os itens com classificação excelente, com exceção dos "glicemia" e "paciente em jejum/pós prandial" que foram classificados em "muito ruim".

\section{Discussão}

Diante do que fora exposto nos resultados dessa pesquisa, o índice de incompletude geral foi de $41,47 \%$, classificado como "ruim". Em relação aos índices das categorias, tem-se que "documentos gerais" teve o maior índice de incompletude $(81,86 \%)$, seguido de "documentos obrigatórios" (58,26\%) e "tratamento" (56,94\%), enquanto "identificação do usuário" $(10,36 \%)$ teve o menor índice. Sobre os índices de incompletude dos itens das categorias, os menores registros foram do "nome" e "pressão sistólica", ambos com 0,66\%, seguidos de "data de nascimento", "nome do logradouro" e "pressão diastólica", ambos com 1,31\%, enquanto os maiores índices foram percebidos em toda a categoria "CTPS", cujo item "série" apresentou o maior índice $(97,05 \%)$ do estudo.

Neste estudo, os resultados encontrados evidenciam que o SIS-Hiperdia no município de Marabá, dentro das unidades estudadas, possui uma incompletude significativa sobre os dados registrados nas fichas de cadastro desse programa. Sabe-se que o Hiperdia é organizado com base nos municípios nele cadastrados e objetiva gerar informações cruciais para os gestores em todas as esferas administrativas (a própria unidade de saúde municipal, os gestores estaduais e o MS), podendo ser o perfil epidemiológico de determinada população, o planejamento de implementação de medidas preventivas para HAS e DM, e até 
mesmo a verificação de presença ou ausência de fatores de risco ou comorbidades (Reis \& Cesarino, 2016). A importância do preenchimento desses dados é, com embasamento em informações científicas, gerar melhoria da qualidade de vida dos indivíduos e valorização dos trabalhadores da atenção básica com ações resolutivas (Alves et al., 2011).

Outra justificativa para o preenchimento geral ter sido classificado como ruim é atribuída aos profissionais de saúde que lidam com o preenchimento da ficha de cadastro. Tem-se que os dispositivos tecnológicos de coleta de dados vêm sendo desenvolvidos longe do contexto onde vão ser utilizados e já chegam às unidades de saúde prontos, através de normas técnicas determinadas pela gestão dos sistemas de informação, sem levar em consideração as percepções dos profissionais responsáveis pelo atendimento das demandas dos usuários. Assim, quando se pensa na contribuição das Tecnologias da Informação e Comunicação em Saúde (TICSs) na produção das ações cuidadoras, é necessário ir além das propostas dos equipamentos e saberes técnicos colocados, e pensar nas relações humanas que são construídas durante o processo de cuidar em saúde (Evangelista, Guimarães \& Leal, 2013).

Sobre o índice de incompletude das categorias, corroborando com esta pesquisa, um estudo realizado no Paraná em 2008 evidenciou que a categoria "documentos gerais" teve o maior índice de não preenchimento (91,06\%), contudo, esses dados não são obrigatórios para o processamento final dos cadastros, sendo essa uma das possíveis justificativas para essa ausência (Oliveira \& Palha, 2008).

Sabe-se também que a informação em saúde é encarada como a principal ferramenta para a construção de uma atenção de qualidade; os discursos apresentados neste campo afirmam que a ausência e a baixa qualidade dos dados são uma das principais causas para o não funcionamento, ou, falhas das políticas de saúde (Merhy \& Chakkour, 2002). Por isso, também é preocupante a incompletude de dados nas categorias "documentos obrigatórios" e "tratamento" serem uma das principais nesse estudo, pois essas omissões prejudicam a elaboração de mais estudos epidemiológicos e dificultam o planejamento de políticas públicas de saúde locais para a população (Zillmer et al., 2010).

A respeito da incompletude dos itens das categorias, os estudos sobre melhores preenchimentos estavam de acordo com os achados nessa pesquisa, pois o nome do paciente, cujo índice de incompletude foi 0,7\% (Correia, 2014), e nome de logradouro, com apenas $0,5 \%$ dos registros ausentes (Zillmer et al., 2010), também foram itens bem preenchidos. Já em relação aos preenchimentos incompletos, foram encontrados resultados diferentes dos verificados nessa pesquisa, em um estudo no Rio Grande do Sul, constataram-se as maiores porcentagens de incompletude em tratamento não medicamentoso (97\%) e no cartão nacional de saúde (95,6\%) (Zillmer et al., 2010), ao passo que em Alagoas, o item com maior incompletude foi glicemia capilar $(66,8 \%)$ (Correia, Padilha, \& Vasconcelos, 2014).

\section{Conclusão}

Considera-se, portanto, que os objetivos propostos nesta pesquisa foram alcançados, tendo em vista que as incompletudes observadas apontam para a necessidade de políticas públicas que fomentem o preenchimento desta ficas em sua totalidade. As análises, discussões e inferências originadas a partir desta pesquisa indicam a necessidade de continuidade do processo de aprimoramento de pesquisas referentes ao tema, bem como produziram contribuições efetivas junto ao contexto atual para elucidação do panorama do SIS-Hiperdia no Município de Marabá, servindo ainda como fonte de dados para futuras ações e/ou estratégias a serem implementadas. Sugere-se, para tanto, a política extensionista entre Secretaria Municipal de Saúde e Instituições de Ensino Superior locais, no intuito de somarem esforços para conscientização e treinamento dos profissionais que preenchem tais fichas e/ou sistemas.

Como fator limitante do estudo, tem-se que somente três UBS's foram analisadas e que as fichas cadastrais nem sempre estavam preenchidas em sua totalidade, podendo, também, terem sido preenchidas com erros. Todavia, é válido salientar que 
estudos como este são fundamentais para o aprimoramento do programa HIPERDIA na cidade de Marabá, sendo importante compreender o perfil dos pacientes de doenças crônicas para que ações educacionais em saúde possam ser colocadas em prática no município.

Ademais, ainda são necessárias mais pesquisas que demostrem a incompletude dos dados do programa Hiperdia em outras localidades do país e estudos que apresentem os impactos desta falta de preenchimento para a população atendida nas unidades de saúde estudadas.

Espera-se que estes achados incentivem o preenchimento de maneira adequada das fichas de atendimento, tendo como meta a maior divulgação de informações que possam subsidiar a elaboração de políticas públicas integradas e intersetoriais para a área da estratégia em saúde da família, e que efetivamente promovam a saúde e a qualidade de vida nesta região do país, assim como no Brasil.

\section{Referências}

Alves, W., Valle, D. C., Santos, C., Luiz, A., Braga, D. S., Sc, M., Mary, A., Tinoco, M., Rosas, F., \& Sc, D. (2011). Revisão Hiperdia , Sistema de Informação em Saúde, prática de enfermagem na Atenção Básica. Enfermagem Brasil, 10(June 2010), 119-125.

Correia, L. O. dos S., Padilha, B. M., \& Vasconcelos, S. M. L. (2014). Completitude dos dados de cadastro de portadores de hipertensão arterial e diabetes mellitus registrados no Sistema Hiperdia em um estado do Nordeste do Brasil. Ciencia e Saude Coletiva, 19(6), 1685-1697. https://doi.org/10.1590/141381232014196.02842013

Dolzane, R. da S., \& Schweickardt, J. C. (2020). Atenção básica no Amazonas: provimento, fixação e perfil profissional em contextos de difícil acesso. Trabalho, Educação e Saúde, 18(3). https://doi.org/10.1590/1981-7746-SOL00288

Evangelista, A. da S., Guimarães, M. C., \& Leal, M. (2013). Fluxos de Informação no SisHiperDia: um estudo de caso . Revista Eletrônica de Comunicação, Informação e Inovação Em Saúde, 7(4), 1-14. https://doi.org/10.3395/reciis.v7i4.873pt

Feitosa, I., \& Pimentel, A. (2016). Hiperdia: Práticas de Cuidado em uma Unidade de Saúde de Belém, Pará. Revista Do NUFEN, 8(1), 13-30. http://pepsic.bvsalud.org/scielo.php?script=sci_arttext\&pid=S2175-25912016000100003

Henriques, O. (2020). SMS: Programa Hiperdia mantem entrega de medicamentos durante a pandemia - Prefeitura de Marabá - Pa. Prefeitura de Marabá https://maraba.pa.gov.br/sms-programa-hiperdia-mantem-entrega-de-medicamentos-durante-a-pandemia/

Instituto Brasileiro de Geografia e Estatística. (2019). IBGE - Cidades e Estados. IBGE. https://www.ibge.gov.br/cidades-e-estados/pa/maraba.html

Instituto Brasileiro de Geografia e Estatística. (2020). Marabá (PA) - Cidades e Estados 2020. IBGE. https://www.ibge.gov.br/cidades-e-estados/pa/maraba.html

Lopes, R. D., \& Harrington, R. A. (2015). Compreendendo a Pesquisa Clínica. In AMGH Editora (Issue 1).

Melo, G. B. T., \& Valongueiro, S. (2015). Incompletude dos registros de óbitos por causas externas no Sistema de Informações sobre Mortalidade em Pernambuco, Brasil, 2000-2002 e 2008-2010*. Epidemiologia e Serviços de Saúde, 24(4), 651-660. https://doi.org/10.5123/S1679-49742015000400007

Merhy, E. E., \& Chakkour, M. (2002). Em busca de ferramentas analisadoras das tecnologias em saúde: a informação e o dia a dia de um serviço, interrogando e gerindo trabalho em saúde. In Agir em Saúde: um desafio para o público (pp. 130-150). https://digitalrepository.unm.edu/lasm_pt/326

Ministério da Saúde. (2008). Hiperdia - Sistema de Cadastramento e Acompanhamento de Hipertensos e Diabéticos. Ministério Da Saúde, 2, 1-104. http://tabnet.datasus.gov.br/cgi/hiperdia/cnv/hddescr.htm\#origem

Ministério da Saúde. (2017). Portaria No 2.436. Ministério Da Saúde. https://bvsms.saude.gov.br/bvs/saudelegis/gm/2017/prt2436_22_09_2017.html

Novaes, A. H., Duarte, F., Riveiro, L. O., \& Santos, T. E. (2019). Metodologia Cientifica Teoria e aplicação na educação a distância. In Journal of Chemical Information and Modeling (Vol. 53, Issue 9). http://portais.univasf.edu.br/dacc/noticias/livro-univasf/metodologia-cientifica-teoria-e-aplicacao-na-educacao-adistancia.pdf

Oliveira, C. A. de, \& Palha, P. F. (2008). Sistema De Informações Hiperdia, 2002-2004, Adequacão Das Informações. Cogitare Enfermagem, 13(3). https://revistas.ufpr.br/cogitare/article/view/12992

Prates, E. J. S., Souza, F. L. P. de, Prates, M. L. S., Moura, J. P. de, \& Carmo, T. M. D. (2020). Perfil clínico-epidemiológico de portadores de diabetes e hipertensão arterial associada. Revista de Enfermagem UFPE on Line, 14. https://doi.org/10.5205/1981-8963.2020.244110

Reis, A. F. N., \& Cesarino, C. B. (2016). <b> Fatores de risco e complicações em usuários cadastrados no hiperdia de São José do Rio Preto/Risk factors and complications among patients registered in the hiperdia in São José do Rio Preto <b>. Ciência, Cuidado e Saúde, 15(1), 118-124. https://doi.org/10.4025/cienccuidsaude.v15i1.24235

Silva, P. A. B., Silva, L. B., Santos, J. F. G., \& Soares, S. M. (2020). Brazilian public policy for chronic kidney disease prevention: challenges and perspectives Revista de Saude Publica, 54, 86. https://doi.org/10.11606/s1518-8787.2020054001708

Soeiro, V. M. da Si., Coimbra, L. C., Aquino, D. M. C. de, Goiabeira, Y. N. L. de A., Viana, L. D. S., \& Ramos, É. L. D. A. (2019). Perfil dos pacientes 
Research, Society and Development, v. 10, n. 9, e37110918040, 2021

(CC BY 4.0) | ISSN 2525-3409 | DOI: http://dx.doi.org/10.33448/rsd-v10i9.18040

acompanhados pelo SIS-HiperDia em um estado do nordeste brasileiro. Arquivos de Ciências Da Saúde, 26(1), 28. https://doi.org/10.17696/23183691.26.1.2019.1230

Sousa, A., \& Costa, A. (2020). Hiperdia: Programa Para a Melhoria Do Controle Dos Pacientes Com Hipertensão Arterial E Diabetes Mellitus Da Estratégia Da Saúde Da Família Do "Santinho I E Ii” Em Barras-Piaú Hiperdia: Program To Improve the Control of Patients With Arterial Hypertensio. UNASUS

Sousa, N., Lima, J., Teixeira, T., Linhares, C., Montes, J. V., \& Marques, J. V. (2019). Fatores De Risco E Complicações Em Diabéticos/Hipertensos Cadastrados No Hiperdia. SANARE - Revista de Politicas Públicas, 18(1), 31-39. https://doi.org/10.36925/sanare.v18i1.1303

Zillmer, J. G. V., Schwartz, E., Muniz, R. M., \& de Lima, L. M. (2010). Avaliação da completude das informações do Hiperdia em uma unidade básica do sul do Brasil. Revista Gaúcha de Enfermagem / EENFUFRGS, 31(2), 240-246. https://doi.org/10.1590/s1983-14472010000200006 